\title{
Effects of Exposure to a Weak Extremely Low Frequency Electromagnetic Field on Daytime Sleep Architecture and Length
}

\author{
Vladimir B. Dorokhov, PhD ${ }^{1,2}$, Anton I. Taranov ${ }^{1}$, Anna M. Narbut ${ }^{3}$, Dmitry S. Sakharov ${ }^{1}$, Svetlana S. Gruzdeva ${ }^{1}$, \\ Olga N. Tkachenko, PhD'1, Gleb N. Arsen'ev' ${ }^{1}$, llya S. Blochin ${ }^{1,2}$, Arcady A. Putilov, PhD ${ }^{1}$ \\ 'Laboratory of Sleep/Wake Neurobiology, Institute of Higher Nervous Activity and Neurophysiology, Russian Academy of Sciences, Moscow, Russia \\ 2Sleep \& Wake Neurotech Center, Skolkovo, Russia \\ ${ }^{3}$ Department of Nervous Diseases, Institute of Professional Education, Sechenov First Moscow State Medical University, Moscow, Russia
}

Received: November 29, 2019

Revised: December 18, 2019

Accepted: December 18, 2019

Correspondence

Arcady A. Putilov, PhD

Laboratory of Sleep/Wake Neurobiology, Institute of Higher Nervous Activity and

Neurophysiology, Russian Academy

of Sciences, Moscow,

11 Nipkowst., Berlin 12489, Germany

Tel +49-30-53674643

Fax +49-30-53674643

E-mail putilov@ngs.ru

\section{ORCID}

Vladimir B. Dorokhov

https://orcid.org/0000-0003-3533-9496 Anton I. Taranov

https://orcid.org/0000-0003-4905-8249

Anna M. Narbut

https://orcid.org/0000-0003-2026-5199

Dmitry S. Sakharov

https://orcid.org/0000-0001-9333-586X

Svetlana S. Gruzdeva

https://orcid.org/0000-0002-5647-5684

Olga N. Tkachenko

https://orcid.org/0000-0002-5100-8980

Gleb N. Arsen'ev

https://orcid.org/0000-0003-3723-7354

Ilya S. Blochin

https://orcid.org/0000-0003-1083-4255

Arcady A. Putilov

https://orcid.org/0000-0003-2779-9046
Background and Objective Human brain appears to be able to absorb, detect, and respond to low-level extremely low-frequency electromagnetic fields (ELF EMF). Controlled laboratory studies on human sleep under exposure to such fields are scarce. Only sleep-disturbing effects on nighttime sleep were reported for frequencies of $50 / 60 \mathrm{~Hz}$, while lower frequencies (i.e., below $20 \mathrm{~Hz}$ ) have not been tested. These frequencies overlap with the frequency range of the electroencephalographic (EEG) signal, and sleep researchers utilized the specific frequency patterns $(1-15 \mathrm{~Hz})$ for subdivision of the sleep-wake state continuum into wake and sleep stages. In particular, the deepest sleep stage (N3) is characterized by slow-wave EEG activity (1-4 Hz) and serves as an electrophysiological indicator of sleep restorative function. We examined the effects of exposure to a low-level ELF EMF on sleep architecture in afternoon naps.

Methods Ten polysomnographic sleep characteristics obtained during two naps of 23 healthy volunteers, either with or without exposure to a $1 \mathrm{~Hz} / 0.004 \mu \mathrm{T}$ electromagnetic field, were compared.

Results The effect of the $1 \mathrm{~Hz} / 0.004 \mu \mathrm{T}$ electromagnetic field exposure on amount of stage N3 was not significant despite the overlap of this intervention frequency with the frequency of slow waves. However, the total duration of sleep was significantly increased due to a significant increase of amount of stage N2. Thus, the exposure to an extremely slow $(1 \mathrm{~Hz})$ electromagnetic field did not reveal any sleep-disturbing effects. Instead, total duration of sleep increased due to increase of N2 amount.

Conclusions A sleep-promoting action of exposure to the low-level $1 \mathrm{~Hz}$ electromagnetic field cannot be excluded.

Sleep Med Res 2019;10(2):97-102

Key Words Extremely low-frequency electromagnetic fields, Post-lunch dip, Daytime sleep quality, Slow wave sleep, Sleep stages.

\section{INTRODUCTION}

Life has evolved in an environment with exposure to extremely low-frequency electromagnetic fields (ELF EMF) of low-level intensity from natural sources, such as magnetic activity of the sun and fields from the earth. The total field intensity produced by sunspots and geomagnetic field in temperate latitudes are equal to $0.3 \mathrm{~T}$ and $50 \mu \mathrm{T}$, respectively. The human brain appears to be able to absorb, detect, and respond to low-level ELF EMF [1]. Over the last 140 years, permanently increasing exposures to the man-made sources have been brought, e.g., from generation, transmission, and use of electricity for power, heating, and lighting. [2] The strengths of fields from all man-made sources can exceed those from natural sources by several orders of magnitude. It was estimated [3] that a modern time long-term exposure to EMF can reach several tenths of $\mu \mathrm{T}$. For example, in such densely populated country as the Netherlands app. 23000 of the 7 million dwellings are situated within the $0.4 \mu \mathrm{T}$ magnetic 
field zone [4].

The studies of the effects of EMF on sleep architecture provided results that are often inconclusive and/or contradictory. For example, the analysis of epidemiological data on sleep quality collected during 10 years in the area surrounding a short-wave (6-22 MHz) broadcasting station, revealed a sleep-disturbing response to EMF field exposure, but in poor rather than good sleepers [5]. Since the effects might be mediated by either electro-magnetic radiation at discrete frequencies or by broadband white noise across the ELF spectrum, such epidemiological findings raise questions regarding whether there is a causal link between ELF and reported effects on human sleep (for more details on these discussions the review by Ohayon et al. [6]).

To date, there have been few controlled laboratory studies on sleep under ELF EMF produced in a narrow frequency range. They were inspired by the concerns about exposure to EMF generated by the electrical power grid and electrical devices with a frequency of $50 \mathrm{~Hz}$ (e.g., in Europe) or $60 \mathrm{~Hz}$ (e.g., in North America). For instance, in a double-blind placebo-controlled study of 18 healthy volunteers Åkerstedt et al. [7] reported that night sleep was impaired by exposure to a $50 \mathrm{~Hz} / 1 \mu \mathrm{T}$ EMF, but all changes in sleep characteristics were still within a normal range. In the study with between-subjects design reported by Graham and Cook [8], continuous, intermittent (1-h off/1-h on), and sham exposure of 7-9 healthy men to a $60 \mathrm{~Hz} / 28.3 \mu \mathrm{T}$ magnetic field was associated with an altered sleep architecture in one of three (intermittent) exposure group.

It remains untested experimentally whether a low-level intensity ELF EMF with profoundly lower than $50-60 \mathrm{~Hz}$ frequencies (e.g., between $1 \mathrm{~Hz}$ and $20 \mathrm{~Hz}$ ) may interfere with human sleep characteristics. These frequencies are of special interest because they are typical for natural ELF EMF and, in the same time, they overlap with the frequency range of the electroencephalographic (EEG) signal recorded from the surface of human scalp. The specific frequency patterns emitted by the brain in the range between $1 \mathrm{~Hz}$ and $20 \mathrm{~Hz}$ were utilized by sleep researchers for subdivision of the sleep-wake state continuum into wake and sleep stages. Namely, the low frequency activity $(<10 \mathrm{~Hz})$ and the sleep spindle frequency activity (app. $12-15 \mathrm{~Hz})$ are two silent features of non-rapid eye movement (NREM) sleep. They serve for distinguishing between stages N1, N2, and N3 of NREM sleep [9] and as markers of the underlying sleep-wake regulating processes [10]. In particular, the deepest stage of sleep, $\mathrm{N} 3$, is characterized by slow waves in 1-4 $\mathrm{Hz}$ frequency range. It is believed that amplitude of these waves reflects intensity of the process of payment of sleep debt accumulated during preceding wakefulness [10].

Therefore, we examined whether an exposure to a low-level intensity $1 \mathrm{~Hz}$ EMF can increase amount of this and other stages of NREM sleep.

A rather big proportion of daytime working people can benefit from afternoon nap [11] that regarded a potent behavioral strategy minimizing sleepiness, fatigue, and impairments of cognitive and physical functioning [12-14]. In general, the conditions of the today daytime environment are far from being optimal for napping (e.g., due to high level of environmental noise, absence of a comfortable bedroom near a working place, etc.). Therefore, it is of both practical and theoretical importance to determine whether efficacy of nap can be increased by exposure to low-level $1 \mathrm{~Hz}$ EMF that falls in the frequency range of slow waves $(1-4 \mathrm{~Hz})$ generated by the brain during the deepest sleep stage. To our knowledge, such a possibility has not been yet experimentally examined.

Consequently, we hypothesized that, due to the overlap of the frequency of this field with the frequency of brain waves, such an intervention might promote stage N3 and other NREM sleep stages in a 50-min afternoon nap.

\section{METHODS}

Study volunteers (23, 19 females) were recruited among students of Moscow Universities and young researchers. The exclusion criteria included reporting age of 18 years or younger, pregnancy or breastfeeding, colds during the previous month, involvement in shift or night work, crossing several meridians during the previous month, irregular sleep-wake schedule (i.e., more than 1-h difference in bedtimes throughout the preceding week) or frequent sleep deprivation (i.e., at least, one case of total or partial sleep deprivation in the previous week). The recruited volunteers denied history of mental or sleep disorder and did not complain about poor physical condition and functioning. Majority of them were familiar with the laboratory environment and experimental napping procedure because they previously participated in a larger nap study [15].

Each participant was informed in detail about the experimental procedures and gave his/her written consent. The study protocol was approved by the Ethics Committee of the Institute of Higher Nervous Activity and Neurophysiology (No. 046/19), and all experimental procedures were performed in accordance with the ethical standards laid down in the Declaration of Helsinki.

The participants visited twice the sleep laboratory at the Institute of Higher Nervous Activity and Neurophysiology for having two afternoon napping attempts with, at least, 1-week interval between the attempts. Each visit lasted for less than 2 hours (between 1 p.m. and 3 p.m.). The participants were randomly assigned to either sham or $1 \mathrm{~Hz} / 0.004 \mu \mathrm{T}$ EMF exposure condition. They were uninformed about an exposure condition and order, and approximately a half of them was exposed to $1 \mathrm{~Hz}$ EMF during the first napping attempt. Thus, the experimental protocol consisted of two 2-h visits in the sleep laboratory divided by, at least, 1-week interval between 1-h afternoon napping attempts under randomly assigned exposures, either sham then 
treatment condition or treatment then sham condition. The conditions did not differ on the number of female participants in either follicular or luteal phase of their menstrual cycle.

After a brief pre-nap interview, the 8-item Epworth Sleepiness Scale (ESS) [16] was administered and then the electrodes for polysomnographic recordings were applied. During the electrodes' application procedure, the participant was lying in bed in the sleep laboratory under dim light ( $<10$ lux). He/she was instructed to try to nap after light off for the following $50 \mathrm{~min}$ and, after hearing the awakening signal at the 50th minute, to remain lying in bed, already without sleep, for the final $10 \mathrm{~min}$ of resting polysomnographic recordings.

A researcher switched off the light using a remote light controller, and the recording started with the first 10-min interval without any interventions. For the next $40 \mathrm{~min}$ (until the signal asking for awakening), an EMF emitting device (the ELF EMF generator "EcoSleep CUBE" manufactured by Center of Neurotechnology of Sleep and Wakefulness, Skolkovo Innovation Center, Moscow, Russia; certified by the State standard GOST R0159555 from 15. 12. 2017) was set by a researcher either on or off. This device was placed at the distance of $700 \mathrm{~mm}$ from the participant's head to allow the generation of the $0.004 \mu \mathrm{T}$ EMF around the head. It was unseen from the bed, and, during any of two napping attempts, the participant cannot recognize whether it was switched on or off. An alternating magnetic field (inductance $\mathrm{L}=0.5 \mu \mathrm{H}$ with the resistance $\mathrm{R}=15 \Omega$ ) was generated by the bifilar planar spiral coils (a coil diameter is 50 $\mathrm{mm}$ ). The highest possible voltage of the field inductor was approximately $3 \mathrm{~V}$. The current had the form of square $1 \mathrm{~Hz}$ pulses with the duty factor equal to 0.5 . To calculate intensity of EMF at the distance of $700 \mathrm{~mm}$ from the device, the field parameters were measured with the BE-METP-AT-002 ("indicator of parameters of electric and magnetic fields," verification certificate №AA 3442920/07356, OOO NTM-Zaschita, Moscow, Russia). The intensity of EMF in the center of inductor $\mathrm{B}_{0}$ was $22 \mu \mathrm{T}$. The field $\mathrm{B}$ induced at the distance $\mathrm{r}$ was calculated as:

$$
\mathrm{B}=\mathrm{B}_{0} \times\left(\mathrm{r}_{0} / \mathrm{r}\right)^{2}
$$

where $r_{0}$ was equal to $10 \mathrm{~mm}$. Thus, on the distance of 700 $\mathrm{mm}, \mathrm{EMF}$ intensity was found to be equal to $0.004 \mu \mathrm{T}$.

The polysomnographic recordings were performed via a 16-channel wireless system ("Neuropolygraph 24," Neurotech, Taganrog, Russia). A standard monitoring montage was used for polysomnographic recordings, namely, the EEG channels, one chin electromyogram channel, and two electro-oculogram channels. All electrodes were placed in accord with the international 10-20 system of electrode placement. For providing a possibility of conventional sleep stage scoring, the placement included a central channel referenced to an ear mastoid site (C4-M1), a central channel referenced to an ear mastoid site (C3-M2), an occipital channel referenced to an ear mastoid site (O1-M2), and an occipital channel referenced to an ear mastoid site (O2-M1). The recorded signals were conditioned by the high-pass, low-pass, and notch filters (frequencies of 0.5, 35, and $50 \mathrm{~Hz}$, respectively). The signals were sampled and stored on a hard disc with a frequency of $500 \mathrm{~Hz}$. Conventional scoring procedure [8] was performed visually on 30 epochs of each 60 -min recording by two experienced scorers. They were uninformed about an exposure condition. Each record was scored by the scorers independently, the initial disagreement varied, depending upon a stage, from $80 \%$ to $95 \%$, and, thereafter, they re-

Table 1. Daytime sleep architecture in sham and $1 \mathrm{~Hz} / 0.2 \mu \mathrm{T}$ electromagnetic fields exposure conditions

\begin{tabular}{|c|c|c|c|c|c|c|c|c|}
\hline \multirow{2}{*}{$\begin{array}{c}\text { Exposure } \\
\text { Min }\end{array}$} & \multicolumn{2}{|c|}{ Sham } & \multicolumn{2}{|c|}{$1 \mathrm{~Hz}$} & \multicolumn{2}{|c|}{ Difference } & \multicolumn{2}{|c|}{ Paired t-test } \\
\hline & Mean & SEM & Mean & SEM & Mean & SEM & $t_{22}$ & $\mathrm{p}$ \\
\hline \multicolumn{9}{|l|}{ Latency to } \\
\hline N1 & 13.09 & 1.85 & 10.63 & 1.29 & 3.16 & 2.62 & 1.033 & 0.313 \\
\hline N2 & 30.87 & 3.89 & 25.17 & 3.48 & 6.66 & 4.52 & 1.485 & 0.152 \\
\hline N3 & 42.26 & 3.37 & 41.30 & 3.67 & 2.13 & 4.62 & 0.233 & 0.818 \\
\hline Sleep length & 35.20 & 3.05 & 41.13 & 2.18 & -7.53 & 3.02 & -2.114 & 0.046 \\
\hline \multicolumn{9}{|l|}{ Amount of } \\
\hline W & 33.89 & 3.10 & 29.11 & 2.84 & 4.78 & 2.87 & 1.665 & 0.110 \\
\hline WASO & 9.09 & 1.85 & 10.24 & 1.57 & -1.15 & 1.75 & 0.658 & 0.518 \\
\hline N1 & 11.30 & 1.58 & 11.28 & 1.75 & 0.02 & 2.23 & 0.010 & 0.992 \\
\hline $\mathrm{N} 2$ & 7.83 & 1.20 & 11.87 & 1.54 & -4.04 & 1.70 & -2.378 & 0.027 \\
\hline N3 & 6.44 & 1.77 & 7.17 & 1.83 & -0.74 & 2.47 & -0.299 & 0.768 \\
\hline $\mathrm{R}$ & 0.54 & 0.54 & 0.57 & 0.40 & -0.02 & 0.69 & -0.031 & 0.975 \\
\hline
\end{tabular}

N1, N2, N3, and R: sleep stages; W: wakefulness; WASO: W after sleep onset; Sleep length: time interval between the first and last occurrence of any sleep stage; Mean and SEM: sample-averaged value of a sleep characteristic and standard error of mean. Sham and $1 \mathrm{~Hz}$ : the alternative conditions with the device either off or on between 10th and 50th min of 1-h napping attempt. Values of any sleep characteristic are given in minutes. See the 60-min time courses of W, N1, N2, N3, and R in Fig. 1. 
examined together all intervals with discrepant scores in order to produce consensus scores. The epochs were classified into stages including wakefulness stage (W), REM sleep (R), and three stages of NREM sleep (stage 1 sleep or N1, stage 2 sleep or $\mathrm{N} 2$, and slow-wave sleep or N3). In the present report, 10 traditional polysomnographic characteristics of sleep architecture were analyzed and reported in Table 1.

Statistical analyses were performed with the SPSS 22.0 statistical software package (IBM Corp., Armonk, NY, USA). A Student's paired t-test was applied to reveal pairwise differences between two conditions. Additionally, two-way repeated measure analysis of variances (rANOVAs) were run on 6 10-min intervals of each sleep stage. For each such rANOVA, Mauchly's test was conducted to assess the sphericity and, if necessary, the Greenhouse-Geiser correction was used to adjust the degrees of freedom. To compare number of participants who either entered or did not enter into stages N2 and N3, $\chi^{2}$-test was employed.

\section{RESULTS}

Mean age \pm standard deviation for 23 study participants was $22.09 \pm 4.55$. Interestingly, after a napping attempt more than a half of them denied that they slept during the 50-min interval, while sleep, at least, N1 (stage 1 sleep) for, at least, one min was detected during any of napping attempts $(n=46)$ as suggested by the results of scoring the polysomnographic recordings. The majority of those who reported they were able to fall asleep usually claimed that, at the 50th min (after hearing the awakening signal), they wish to further remain asleep. Indeed, the polysomnographic data suggested that, at least, one of the last 20 scoring epochs (minutes from 51st to 60th, after hearing the awakening signal) was classified as a sleep stage in more than a half of all recordings.

However, 5 study participants did not reach stage N2 (stage 2 sleep) in the sham condition. Two of these 5 reached this stage in the treatment condition. Nevertheless, $\chi^{2}$-test did not reveal a statistically significant difference between conditions $\left(\chi^{2}=0.605, \mathrm{p}=0.437\right)$. Almost a half of study participants did not reach stage $\mathrm{N} 3$, the deepest slow wave sleep stage (10 vs. 11 in the sham vs. treatment condition, respectively). The difference between conditions in number of participants without and with N3 also did not reach a statistically significant level $\left(\chi^{2}=\right.$ $0.088, \mathrm{p}=0.767)$.

Among 10 pairwise comparisons of nap characteristics shown in Table 1, a significant increase of sleep length under exposure to $1 \mathrm{~Hz} / 0.004 \mu \mathrm{T}$ EMF was revealed by paired t-test. This increase occurred mostly due to a significant increase in amount of N2. An amount of N3 did not change significantly in the 1 $\mathrm{Hz} / 0.004 \mu \mathrm{T}$ EMF exposure condition compared to the sham condition (Table 1).

Similarly, N2 was the only sleep stage for which two-way rANOVA with repeated measures "condition" (either without or with exposure to $1 \mathrm{~Hz} / 0.004 \mu \mathrm{T}$ EMF between the 10th and 50 th min of napping attempt) and "10-min time interval" (6 10min intervals) yielded a significant main effect of "condition" (Fig. 1). Such main effect was significant for neither N1 nor N3 nor R nor W (Fig. 1).

In comparison of the pairs of polysomnographic measures representing the first and second napping attempts, a significant influence of order of exposure to $1 \mathrm{~Hz}$ EMF was not found

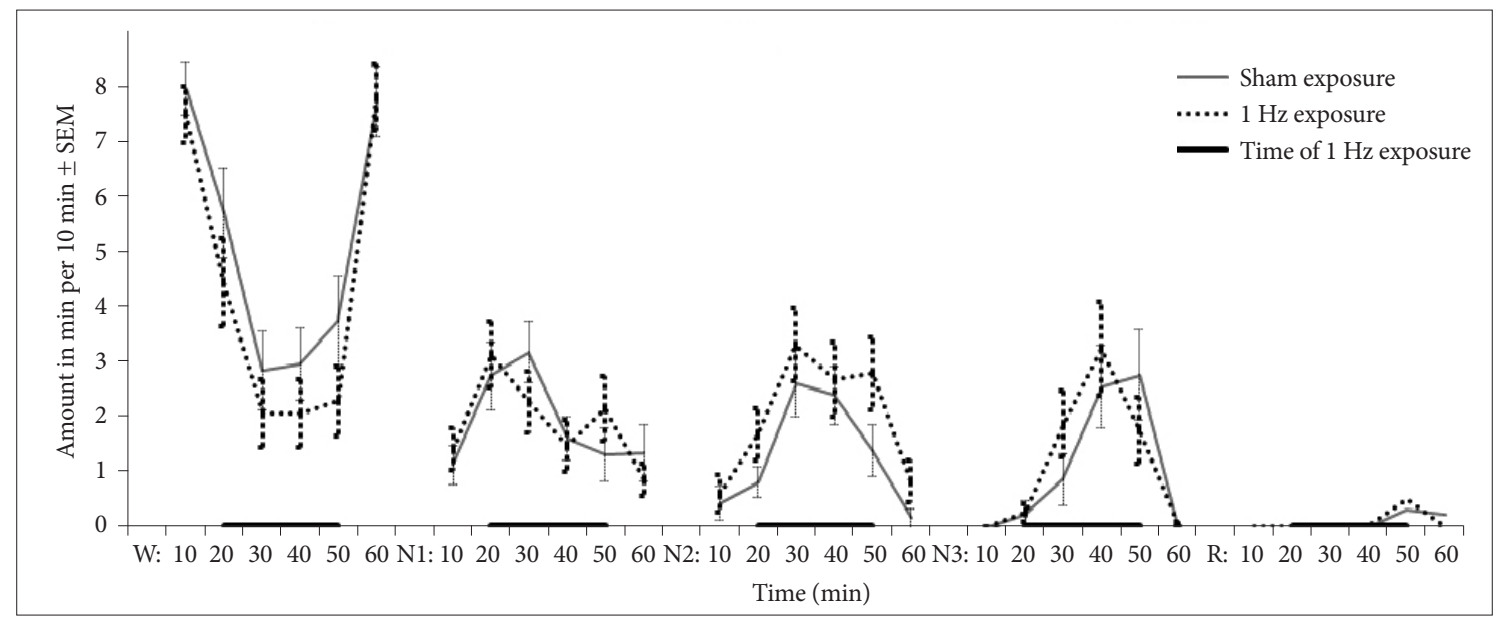

Fig. 1. Time courses of amount of wakefulness and sleep stages in sham and $1 \mathrm{~Hz} / 0.004 \mu \mathrm{T}$ electromagnetic fields (EMF) exposure conditions. Each polysomnographic record of napping attempt was divided into 610 -min intervals; 10, 20, 30, 40, 50, and 60 min: amount of stage for each of $610-\mathrm{min}$ intervals of 1-h (60-min) napping attempt with the signal of waking up at the 50th min (30-s scoring epochs 1-20, 21-40, 41-60, 61-80, 81-100, and 101-120); W: wake state; N1, N2, N3, and R: sleep stages. Mean amounts of stages per 10 min and standard error of mean (SEM) were obtained by applying two-way rANOVAs with repeated measures "condition" (either without or with exposure to $1 \mathrm{~Hz} / 0.004 \mu \mathrm{T}$ EMF between the 10th and 50th min of napping attempt) and "10-min time interval" (6 10-min intervals). The rANOVA yielded a significant main effect of "condition" $\left(F_{1,22}=5.655, p=0.027\right)$ for N2 only, with the effect size (partial eta squared) of 0.341 . See also averaged over the whole napping attempt amounts of sleep stages in Table 1. rANOVAs: repeated measure analysis of variances. 
( $\mathrm{p}>0.05$ for all 10 paired t-tests).

The difference between ESS scores obtained prior napping attempts in two (sham and treatment) exposure conditions was very small and non-significant (mean \pm standard deviation equal to $-0.087 \pm 1.756, \mathrm{t}_{22}=-0.238, \mathrm{p}=0.814$ ). The Spearman's rank correlation coefficient between scores for these two conditions attained the value of 0.921 ( $\mathrm{p}<0.001)$. Mean score \pm standard deviation was $8.87 \pm 4.67$. In accord with cut-points suggested by Johns [16] and Aurora et al. [17], only 4 of 23 participants of the study had ESS score of 11 or higher indicating either mild (11 and 12) or moderate excessive daytime sleepiness (13 and 15). However, this small group did not differ significantly from the remaining sample on the polysomnographic sleep characteristics.

\section{DISCUSSION}

Controlled laboratory studies on human sleep under lowlevel intensity ELF EMF are scarce, and sleep-disturbing rather than sleep-promoting effects were reported in the experiments examining frequencies generated by the electrical power grid and electrical devices $(50$ and $60 \mathrm{~Hz}$ ) $[7,8]$. Since lower frequency range $(1-20 \mathrm{~Hz})$ overlaps with the frequency range of the most powerful spectral components of EEG signal emitted by the brain during NREM sleep, it seems to be of special interest to examine whether a $1 \mathrm{~Hz}$ EMF of close to natural intensity interferes with human sleep. Particularly, one can speculate that the EMF of the particular frequency range $(1-4 \mathrm{~Hz})$ might increase an amount of the deepest sleep stage (N3) distinguished from other stages by high amplitude slow waves and associated with slow wave activity $(1-4 \mathrm{~Hz})$, an indicator of sleep restorative function. In the present pilot study, we tested possibility to increase an amount of $\mathrm{N} 3$ and other NREM sleep stages by exposing 23 healthy volunteers during minutes $10^{-}$ 50th of an afternoon napping attempt to a low-level $(0.004 \mu \mathrm{T})$ 1-Hz EMF. Although amount of N3 remained unchanged in this exposure condition, the total duration of sleep become longer due to increase of N2 amount.

It seems that using a larger sample for further examination of the sleep-promoting effects of exposure to a low-level $1 \mathrm{~Hz}$ EMF on daytime sleep quality is necessary for demonstration of replicability of the revealed effects. Moreover, a possible sleeppromoting rather than sleep-disturbing action of exposure to low-level ELF EMF below $20 \mathrm{~Hz}$ on sleep might be further examined experimentally by comparison of different (lower vs. higher) frequencies (e.g., in the ranges of 1-20 Hz and 33-64 Hz). However, it has to be noted that, if the differential response to lower and higher frequencies has been found, this finding would raise the next question regarding whether this is due to a beneficial effect of lower frequency exposure or due to an adverse effect of higher frequency exposure. Finally, further examination might be also aimed on testing a possibility of reducing latency to $\mathrm{N} 2$ by switching an EMF emitting device in the very beginning of napping attempt rather than on its 10th minute. If such further experimental studies will lend support for the assumption of sleep-promoting rather than sleep-disturbing action of exposure to low-level ELF EMF, such a non-invasive intervention might be recommended for improving condition of afternoon naps aimed on reducing daytime sleepiness and fatigue.

In sum, results of the present pilot study seem to differ from the previously reported results showed that exposure to a lowlevel EMF with frequencies 50 and $60 \mathrm{~Hz}$ has an adverse rather than beneficial effect on human sleep. In contrast, we found that the exposure to a much slower $(1 \mathrm{~Hz})$ EMF did not produce any sleep-disturbing effects. Even more, we observed the increase of total sleep duration due to the increase of amount of $\mathrm{N} 2$. This result suggests a possibility of sleep-promoting action of $1 \mathrm{~Hz} / 0.004 \mu \mathrm{T}$ EMF exposure. A somewhat stronger evidence for such action might be provided by further (confirmation) experiments with samples of a larger size exposed to EMF in frequency range 1-20 Hz and by the new experiments aimed on comparing the effects of low-level EMF in this frequency range with the effects of EMF in higher frequency range, e.g., from 33 $\mathrm{Hz}$ to $64 \mathrm{~Hz}$.

\section{Acknowledgments}

The studies were supported by grants from the Russian Foundation for Basic Research (VBD by grant number 17-36-00025-OGN-MOL-A1 and AAP by grant number 19-013-00424).

The authors thank Ekaterina V. Tiunova and Irina A. Piletskaya form the Moscow State Pedagogical University (Moscow, Russia) for help in conducting the experiments and the authors are very grateful to 23 unpaid volunteers for their participation in the study.

\section{Conflicts of Interest}

The authors have no financial conflicts of interest.

\section{Authors' Contribution}

VBD, AIT, AMN, DSS, SSG, ONT, GNA, ISB, and AAP equally contributed to data collection, analysis, and interpreting the results, and the manuscript was mostly written by AAP.

\section{REFERENCES}

1. Carrubba S, Marino AA. The effects of low-frequency environmentalstrength electromagnetic fields on brain electrical activity: a critical review of the literature. Electromagn Biol Med 2008;27:83-101.

2. Swanson J. Long-term variations in the exposure of the population of England and Wales to power-frequency magnetic fields. J Radiol Prot 1996;16:287-301.

3. Ilonen K, Markkanen A, Mezei G, Juutilainen J. Indoor transformer stations as predictors of residential ELF magnetic field exposure. Bioelectromagnetics 2008;29:213-8.

4. Kelfkens G, Van Wolven J, Pennders R, Stuurman C, Van Aernsbergen L, Delfini G, et al. Costs and benefits of the reduction of magnetic fields due to overhead power lines. In: Kostarakis P, editor. Proceedings of the 2nd International Workshop on Biological Effects of Electromagnetic Fields; 2002 Oct 11; Rhodos, Greece. Ioannina: University of Ioannina;2002. p.309-17.

5. Altpeter ES, Röösli M, Battaglia M, Pfluger D, Minder CE, Abelin T. Ef- 
fect of short-wave (6-22 MHz) magnetic fields on sleep quality and melatonin cycle in humans: the Schwarzenburg shut-down study. Bioelectromagnetics 2006;27:142-50.

6. Ohayon MM, Stolc V, Freund FT, Milesi C, Sullivan SS. The potential for impact of man-made super low and extremely low frequency electromagnetic fields on sleep. Sleep Med Rev 2019;47:28-38.

7. Åkerstedt T, Arnetz B, Ficca G, Paulsson LE, Kallner A. A 50-Hz electromagnetic field impairs sleep. J Sleep Res 1999;8:77-81.

8. Graham C, Cook MR. Human sleep in $60 \mathrm{~Hz}$ magnetic fields. Bioelectromagnetics 1999;20:277-83.

9. Iber C. The AASM manual for the scoring of sleep and associated events: rules, terminology, and technical specification. 1th ed. Westchester, IL: American Academy of Sleep Medicine;2007.

10. Borbély AA, Daan S, Wirz-Justice A, Deboer T. The two-process model of sleep regulation: a reappraisal. J Sleep Res 2016;25:131-43.

11. Putilov AA, Marcoen N, Neu D, Pattyn N, Mairesse O. There is more to chronotypes than evening and morning types: results of a large-scale community survey provide evidence for high prevalence of two further types. Pers Individ Differ 2019;148:77-84.
12. Takahashi M, Nakata A, Haratani T, Ogawa Y, Arito H. Post-lunch nap as a worksite intervention to promote alertness on the job. Ergonomics 2004;47:1003-13.

13. Milner CE, Cote KA. Benefits of napping in healthy adults: impact of nap length, time of day, age, and experience with napping. J Sleep Res 2009; 18:272-81.

14. Caldwell JA, Mallis MM, Caldwell JL, Paul MA, Miller JC, Neri DF; Aerospace Medical Association Fatigue Countermeasures Subcommittee of the Aerospace Human Factors Committee. Fatigue countermeasures in aviation. Aviat Space Environ Med 2009;80:29-59.

15. Shumov DE, Yakovenko IA, Dorokhov VB, Sveshnikov DS, Yakunina EB, Bakaeva ZV, et al. Napping between scylla and charybdis of N1 and N3: latency to N2 in a brief afternoon nap can be reduced by binaural beating. Biol Rhythm Res 2019:1587839.

16. Johns MW. A new method for measuring daytime sleepiness: the epworth sleepiness scale. Sleep 1991;14:540-5.

17. Aurora RN, Caffo B, Crainiceanu C, Punjabi NM. Correlating subjective and objective sleepiness: revisiting the association using survival analysis. Sleep 2011;34:1707-14. 